\begin{tabular}{|c|l|}
\hline Title & A Throughput Evaluation of an Over-Distributed A ntenna Sy stem with Limited Pilot Resources \\
\hline Author(s) & Nakanishi, Yuki; Nishimura, Toshihiko; Ohgane, Takeo; Ogawa, Y asutaka; Ohwatari, Y usuke; Kishiyama, Y oshihisa \\
\hline Citation & $\begin{array}{l}\text { IEICE transactions on communications, E98B(8), 1465-1473 } \\
\text { https:/doi.org/10.1587/transcom.E98.B.1465 }\end{array}$ \\
\hline Issue Date & 2015-08 \\
\hline Doc URL & http://hdl.handle.net/2115/59898 \\
\hline Rights & copyright@2015 IEICE \\
\hline Type & article \\
\hline File Information & e98_b_8_1465.pdf \\
\hline
\end{tabular}

Instructions for use 


\title{
A Throughput Evaluation of an Over-Distributed Antenna System with Limited Pilot Resources*
}

\author{
Yuki NAKANISHI ${ }^{\dagger a)}$, Student Member, Toshihiko NISHIMURA ${ }^{\dagger}$, Takeo OHGANE ${ }^{\dagger b)}$, Members, , \\ Yasutaka OGAWA ${ }^{\dagger}$, Fellow, Yusuke OHWATARI ${ }^{\dagger \dagger}$, and Yoshihisa KISHIYAMA $^{\dagger \dagger}$, Members
}

\begin{abstract}
SUMMARY A distributed antenna system, where the antennas of a base station are spatially distributed throughout the cell, can achieve better throughput at the cell edge than a centralized antenna system. On the other hand, the peak throughput degrades in general because each remote antenna unit has only a few antennas. To achieve both high peak and cell-edge throughputs, we need to increase the total number of antennas. However, this is not easy due to the pilot resource limitation when we use frequency division duplexing. In this paper, we propose using more antennas than pilot resources. The number mismatch between antennas and signals is solved by using a connection matrix. Here, we test two types of connection matrix: signal-distributing and signal-switching. Simulation results show that the sum throughput is improved by increasing the number of antenna elements per remote antenna unit under a constraint on the same number of pilot resources.

key words: distributed antenna system, multiuser MIMO system, throughput, pilot resource
\end{abstract}

\section{Introduction}

Currently, the rapid increase of traffic on mobile communications is considered a serious problem. It is said that if the traffic continues to increase at its current rate, the volume of mobile traffic in 2020s will be at least 1000-fold larger than that in 2010 [2]. To support such a large amount of traffic and realize high speed and large capacity wireless communication in the future, a fifth generation $(5 \mathrm{G})$ system is being discussed as a new mobile communications system.

Multiple-input multiple-output (MIMO) technology needs to be enhanced for a $5 \mathrm{G}$ system. A MIMO system can increase the channel capacity in proportion to the number of transmit and receive antenna elements [3], but in general, the number of antennas at user terminals is difficult to increase due to the hardware restriction. In such a case, we can achieve high system throughput by applying a multiuser MIMO concept [4]-[6] where a base station has many transmit antennas and communicates with several users simultaneously. As an extension of this concept, recently, a very large or massive MIMO system has been proposed that can

\section{Manuscript received December 8, 2014}

Manuscript revised March 3, 2015.

${ }^{\dagger}$ The authors are with the Graduate School of Information Science and Technology, Hokkaido University, Sapporo-shi, 0600814 Japan.

${ }^{\dagger}$ The authors are with NTT DOCOMO, INC., Yokosuka-shi, 239-8536 Japan.

*This paper was partly presented at IEEE PIMRC 2014 [1].

a) E-mail: naka38@m-icl.ist.hokudai.ac.jp

b)E-mail: ohgane@ist.hokudai.ac.jp

DOI: 10.1587/transcom.E98.B.1465 achieve extremely high capacity [7]

Although most massive MIMO concepts are discussed for use in a centralized antenna system (CAS) where antennas of a base station are located at the center of the cell, the concept is theoretically applicable to a distributed antenna system (DAS) [8]-[10] as well. The DAS is known to improve the cell-edge throughput performance. Thus, it is suitable to maintain the fairness of user throughput. Fortunately, a concept of cloud radio access network [11], which is a key technology for $5 \mathrm{G}$, provides a DAS-friendly architecture.

However, the DAS has a disadvantage that the peak throughput tends to be lower than that of the CAS. In the DAS, the antenna elements are distributed to several remote antenna units (RAUs). Thus, the number of antennas per RAU is obviously smaller than the number of antennas at the CAS base station. If the total number of antennas is not enough, each RAU may have only a couple of antennas. Thus, the spatial multiplexing capability becomes small even near the RAU due to the rank deficiency. From this point of view, the massive MIMO concept is suitable for DAS because the number of antennas per RAU also becomes large enough.

Any precoding methods require the channel state information (CSI) per transmit antenna. Therefore, how we obtain the CSI in massive MIMO systems is a big issue. To know the CSI, we send an individual pilot sequence from each transmit antenna. In the massive MIMO scenario discussed by Rusek et al. [12], the total number of user terminals as well as their receive antennas is assumed to be much smaller than the number of antennas at the base station. This means that uplink CSI estimation requires much fewer pilot resources than the downlink one. Therefore, in such a case, time division duplexing (TDD) is considered to leverage the channel reciprocity obtaining the transmit CSI [7], [12], [13].

In the frequency division duplexing (FDD), on the other hand, the channel reciprocity cannot be exploited and the CSI is learned from feedback from user terminals using downlink pilot resources. Thus, a very large number of pilot resources is required to obtain the downlink CSI in massive MIMO scenarios. This is generally unacceptable due to enormous overhead [14], [15].

In the paper, we consider breaking the one-on-one relationship between the transmit antenna and pilot resource and making the total number of transmit antennas exceed 


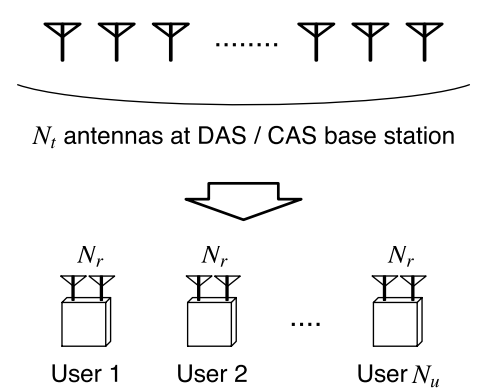

Fig. 1 Multiuser MIMO system.

We denote the channel matrix between the base station and the $k$ th user as $\mathbf{H}^{(k)} \in \mathbb{C}^{N_{r} \times N_{t}}$

$$
\mathbf{H}^{(k)}=\left[\begin{array}{cccc}
h_{11}^{(k)} & h_{12}^{(k)} & \cdots & h_{1 N_{t}}^{(k)} \\
h_{21}^{(k)} & h_{22}^{(k)} & \cdots & h_{2 N_{t}}^{(k)} \\
\vdots & \vdots & h_{i j}^{(k)} & \vdots \\
h_{N_{r} 1}^{(k)} & h_{N_{r} 2}^{(k)} & \cdots & h_{N_{r} N_{t}}^{(k)}
\end{array}\right],
$$

where $h_{i j}^{(k)} \in \mathbb{C}\left(i=1,2, \ldots, N_{r}, j=1,2, \ldots, N_{t}\right)$ expresses the $(i, j)$ th channel response for the $k$ th user. We clearly ignore the time-dispersive channel for the sake of simplicity. However, the following discussion can be applied to multicarrier systems without loss of generality.

We assume that $h_{i j}^{(k)}$ is given by a product of an i.i.d. complex Gaussian random variable (where the mean is zero and the variance is one) and a coefficient $G$ expressing the path loss and shadowing loss. Denoting a path loss constant as $\alpha$ and a random variable for shadowing loss as $\eta$ [dB], which is normally distributed with zero mean and a standard deviation of $\sigma_{s}$, we obtain

$$
G=d^{-\alpha} 10^{-\eta / 10}
$$

where $d$ is a distance from a user terminal to the base station or one of the RAUs [17].

\section{Concept of an Over-Distributed Antenna System}

\subsection{Potential Problem of a Multiuser MIMO DAS}

When the number of total antennas at the base station is limited, each RAU has only a few antennas in the DAS. In a seven-RAUs case, for example, each RAU has only $N_{t} / 7$ antennas. Thus, the user near one of the RAUs can see only a small number of antennas, so rank deficiency may occur as shown in Fig. 2. This is the reason the DAS cannot provide very high peak throughput.

To improve the peak throughput of the DAS, we must increase the number of total antennas at the base station. However, this usually requires pilot resources equal to the number of antennas for channel estimation when we assume FDD systems. Specifically, when we express the number of pilot sequences used for channel estimation as $N_{c}$, $N_{c} \geq N_{t}$ should be ensured. A large number of pilot sequences causes the serious pilot transmission overhead and

\footnotetext{
Our objective in this paper is to compute an upper bound. The impact of control delay should be a future work.
} 


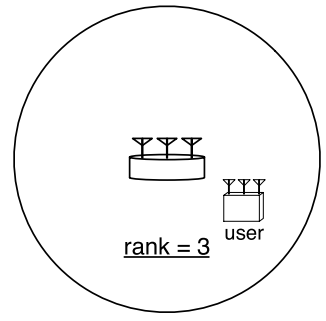

(a) CAS

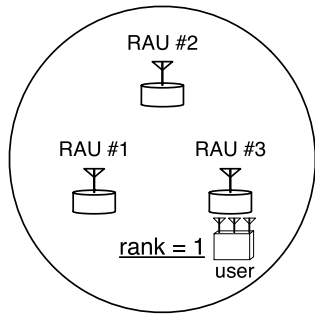

(b) DAS
Fig. 2 Rank difference between CAS and DAS.

degrades transmission efficiency. Thus, it is very difficult to increase the number of antennas per RAU under the constraint of $N_{c} \geq N_{t}$.

\subsection{Over-Distributed Antenna System}

Considering the above problem, we propose the multiuser MIMO DAS with a much larger number of transmit antennas than pilot resources $\left(N_{c}<N_{t}\right)$, i.e., O-DAS. This system is only allowed to use up to $N_{c}$ dimensional channel information at the base station. This means that the number of spatially-multiplexed signals is not larger than $N_{c}$ either. Therefore, the assignment of $N_{c}$ signals to $N_{t}$ transmit antennas must be considered. Here, let us express the RF output signal vector of the central unit as $\mathbf{x}=\left[x_{1}, x_{2}, \ldots, x_{N_{c}}\right]^{T}$ and the output signal vector of the actual antenna elements as $\mathbf{x}^{\prime}=\left[x_{1}^{\prime}, x_{2}^{\prime}, \ldots, x_{N_{t}}^{\prime}\right]^{T}$. These relationships can be expressed by using the connection matrix $\mathbf{T}$ as

$$
\mathbf{x}^{\prime}=\mathbf{T} \mathbf{x}
$$

where $\mathbf{T} \in \mathbb{C}^{N_{t} \times N_{c}}$ indicates a connection status on each output signal from the central unit to transmit antennas. This means that the transmission method and the performance in the O-DAS are determined by the structure of the connection matrix T. In this section, we explain two types of transmission methods and structures of the connection matrix.

\subsubsection{Signal Distributing}

First, we consider the case where the base station cannot acquire or does not use information on the location of user terminals. In the O-DAS (and DAS as well), the RAUs are located apart from each other. Therefore, each user sees only a few RAUs. In other words, the signals transmitted from RAUs on the far side of the cell may not reach the user terminals. This fact implies that the same signal (and the same pilot sequence) among several RAUs can be reused. The signal reuse corresponds to distributing the signal to different RAUs. Thus, we call this transmission type signal distributing.

We explain the concept using the example shown in Fig. 3. Here, the central unit has three RF ports. In general, each port is connected to an individual antenna element as shown in Fig. 3(a). In the signal distributing example, each port is connected to two different antennas as shown

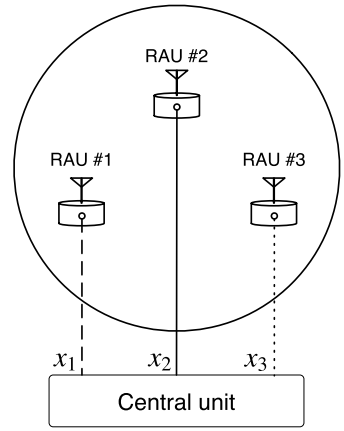

(a) without distributing

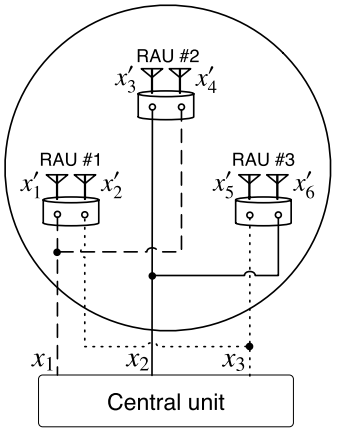

(b) with distributing
Fig. 3 Concept of signal distributing.

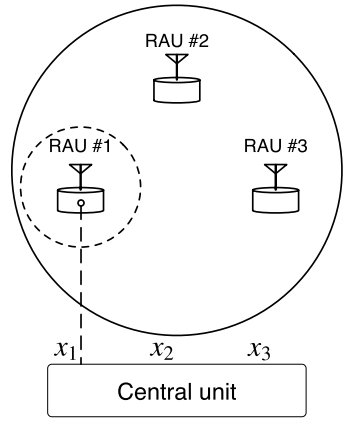

(a) without distributing

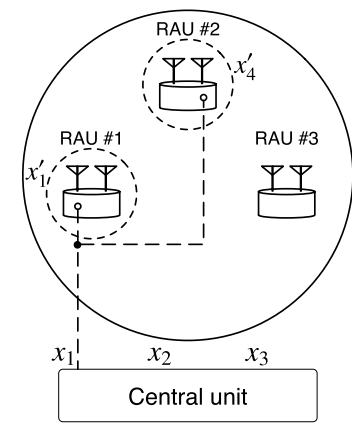

(b) with distributing
Fig. 4 Footprint of RF output port 1.

in Fig. 3(b). This doubles the number of antenna elements without increasing the number of pilot resources.

The effect of signal distributing may be expressed by a footprint (i.e., a coverage area) of each antenna port as shown in Fig. 4. The antennas transmitting distributed signals cover two different circular areas with a single RF output port. This means that the requirement on the user location to receive the signal $x_{1}$ is eased. This may be said to be a very attractive feature of the O-DAS. Although the interference occurs among the distributed pilot signals when we estimate channels, we regard the antennas transmitting the same signal as a virtual antenna and use its effective CSI for transmission, as will be discussed later in Sect. 4.1. If the signal distribution is applied to the CAS, the effective antenna pattern changes and thus is no longer omnidirectional. Note that the transmission power is also distributed. If the power is divided equally, the transmission power per antenna port becomes the inverse of the number of distributed ports connected to the same RF port of the central unit.

The relationship between the RF output ports of the central unit and the actual antenna elements in Fig. 3(b) is expressed as 


$$
\left[\begin{array}{c}
x_{1}^{\prime} \\
x_{2}^{\prime} \\
\vdots \\
x_{6}^{\prime}
\end{array}\right]=\mathbf{T}\left[\begin{array}{l}
x_{1} \\
x_{2} \\
x_{3}
\end{array}\right]=\left[\begin{array}{ccc}
1 / \sqrt{2} & 0 & 0 \\
0 & 0 & 1 / \sqrt{2} \\
0 & 1 / \sqrt{2} & 0 \\
1 / \sqrt{2} & 0 & 0 \\
0 & 0 & 1 / \sqrt{2} \\
0 & 1 / \sqrt{2} & 0
\end{array}\right]\left[\begin{array}{l}
x_{1} \\
x_{2} \\
x_{3}
\end{array}\right] .
$$

Here, $\mathbf{T}$ has only one non-zero element in each row, and the vector norm of each column is normalized. In addition, the same RF port of the central unit is not connected more than once within the same RAU to maintain the omni-directional antenna pattern. Note that the structure of $\mathbf{T}$ is fixed regardless of the locations of user terminals. In the DAS case, $\mathbf{T}$ is given by the identity matrix.

\subsubsection{Signal Switching}

Next, we consider the case where the base station can use information on the location of user terminals. Again, RAUs on the far side of the cell may be insignificant from a signal level viewpoint. Therefore, in this situation, we do not activate such RAUs. Only $N_{c}$ antenna ports are selected from $N_{t}$ ones and connected to the RF ports of the central unit. This concept is explained using the example shown in Fig. 5. Here, we have six antenna ports. On the other hand, three RF ports of the central unit are available. Then, we select three antenna ports and connect them to the central unit. Other antenna ports are disabled. For antenna selection, the user location information is used. The selection process is done on a distance basis with a round robin. Specifically, an arbitrary antenna at the nearest RAU for user 1 is selected first. Then, the selection proceeds to user 2, and so on. When the round is completed, the process is repeated again until the number of selected antennas reaches $N_{c}$.

The relationship between the RF output ports of the central unit and the actual antenna elements in Fig. 5 is expressed as

$$
\left[\begin{array}{c}
x_{1}^{\prime} \\
x_{2}^{\prime} \\
\vdots \\
x_{6}^{\prime}
\end{array}\right]=\mathbf{T}\left[\begin{array}{l}
x_{1} \\
x_{2} \\
x_{3}
\end{array}\right]=\left[\begin{array}{lll}
1 & 0 & 0 \\
0 & 1 & 0 \\
0 & 0 & 0 \\
0 & 0 & 0 \\
0 & 0 & 1 \\
0 & 0 & 0
\end{array}\right]\left[\begin{array}{l}
x_{1} \\
x_{2} \\
x_{3}
\end{array}\right] .
$$

Here, T has only one "1" element in each column, and it appears to be a sparse matrix. In contrast to the signal distributing method, the structure of $\mathbf{T}$ is changed when the locations of user terminals change.

\section{Multiuser MIMO Precoding with Proposed Meth- ods}

In multiuser MIMO systems, one way to prevent the inter user interference (IUI) is precoding. Here, we use block diagonalization (BD) [18]-[20], which is a technique to achieve perfect nulling. To perform precoding at the base station, the channel matrix must be known at the base station. Obviously, the estimated results are affected by signal distributing and signal switching. However, the difference in transmission methods depends on the structure of the connection matrix. Therefore, we can formulate these methods in the same way using $\mathbf{T}$. We describe the precoding procedure in the signal distributing and signal switching step by step.

\subsection{Channel Estimation}

Here we consider an example case using a constant amplitude zero auto-correlation (CAZAC) sequence $\left[p_{1}, p_{2}, \ldots, p_{N_{c}}\right]$ the length of which is $N_{c}$. It is a cyclic shift sequence, thus $N_{c}$ patterns of $N_{c}$ dimensional row vectors are applicable as pilot sequences:

$$
\begin{aligned}
\mathbf{p}_{1} & =\left[p_{1}, p_{2}, \ldots, p_{N_{c}}\right] \\
\mathbf{p}_{2} & =\left[p_{2}, p_{3}, \ldots, p_{1}\right] \\
& \vdots \\
\mathbf{p}_{N_{c}} & =\left[p_{N_{c}}, p_{1}, \ldots, p_{N_{c}-1}\right] .
\end{aligned}
$$

These vectors have a property for the inner product as expressed by

$$
\mathbf{p}_{k} \mathbf{p}_{l}^{H}=\left\{\begin{array}{ll}
N_{c} & l=k \\
0 & l \neq k
\end{array} .\right.
$$

Next, let us describe a channel estimation process with the CAZAC sequences. By using Eq. (6), the matrix consisting of the $N_{c}$ vectors is represented by

$$
\mathbf{P}=\left[\begin{array}{llll}
\mathbf{p}_{1}^{T} & \mathbf{p}_{2}^{T} & \cdots & \mathbf{p}_{N_{c}}^{T}
\end{array}\right]^{T} .
$$

This matrix has a property:

$$
\mathbf{P P}^{H}=N_{c} \mathbf{I}_{N_{c}},
$$

where $\mathbf{I}_{N_{c}}$ denotes the $N_{c}$-dimensional identity matrix.

Fig. 5 Concept of signal switching. 


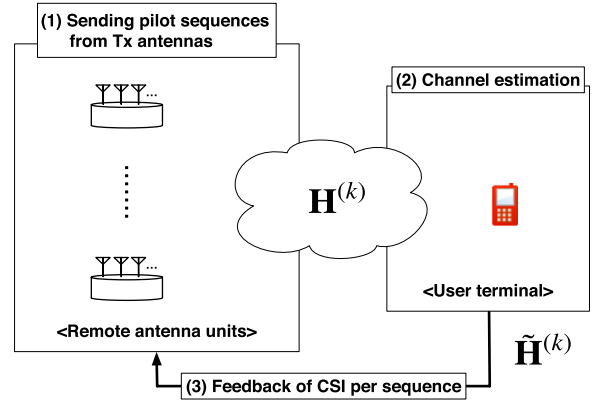

Fig. 6 Channel sounding and feedback process.

The pilot sequences are transmitted from the antenna elements at the base station or RAUs. If we use the signal distributing or signal switching technique, the pilot sequences are distributed in accordance with the connection matrix T. Thus, the pilot sequence matrix $\mathbf{P}^{\prime} \in \mathbb{C}^{N_{t} \times N_{c}}$ transmitted from the actual antenna elements is given by

$$
\mathbf{P}^{\prime}=\mathbf{T P} \text {. }
$$

At the $k$ th user terminal, the received signal sequence corresponding to the pilot sequence duration is written as

$$
\mathbf{Y}^{(k)}=\mathbf{H}^{(k)} \mathbf{T P},
$$

where $\mathbf{Y}^{(k)} \in \mathbb{C}^{N_{r} \times N_{c}}$ is the received signal matrix and the noise is omitted for a simple notation. By multiplying Eq. (11) from the right hand side by $\mathbf{P}^{H}$, we have

$$
\mathbf{Y}^{(k)} \mathbf{P}^{H}=N_{c} \mathbf{H}^{(k)} \mathbf{T} .
$$

Defining estimated channel as $\tilde{\mathbf{H}}^{(k)} \in \mathbb{C}^{N_{r} \times N_{c}}$, we obtain

$$
\tilde{\mathbf{H}}^{(k)}=\frac{1}{N_{c}} \mathbf{Y}^{(k)} \mathbf{P}^{H}=\mathbf{H}^{(k)} \mathbf{T} .
$$

Note that we estimate not the pure channel matrix $\mathbf{H}^{(k)}$ but the effective channel matrix. The estimated channel is fed back to the base station as shown in Fig. 6 .

\subsection{BD Precoding}

BD completely suppresses the IUI by directing nulls to other users. Now, we have an estimated channel matrix $\tilde{\mathbf{H}}^{(k)}$ for $1 \leq k \leq N_{u}$. If the CSI is perfectly estimated, $\tilde{\mathbf{H}}^{(k)}=\mathbf{H}^{(k)} \mathbf{T}$ holds as in Eq. (13).

The $\mathrm{BD}$ precoding matrix $\mathbf{W}_{\mathrm{BD}}^{(k)}$ is calculated on the basis of the estimation results $\tilde{\mathbf{H}}^{(1)}, \ldots, \tilde{\mathbf{H}}^{\left(N_{u}\right)}$, and then the dimension of precoding matrix becomes $N_{c} \times N_{w}$ where $N_{w}=N_{c}-\left(N_{u}-1\right) N_{r}$. The obtained precoding matrix satisfies

$$
\tilde{\mathbf{H}}^{(l)} \mathbf{W}_{\mathrm{BD}}^{(k)}=\mathbf{O} \quad \text { for } \quad l \neq k,
$$

where $\mathbf{O} \in \mathbb{C}^{N_{r} \times N_{w}}$ is the zero matrix. Thus, no IUI is observed at any user terminal. By using Eq. (13), we can rewrite this relationship as

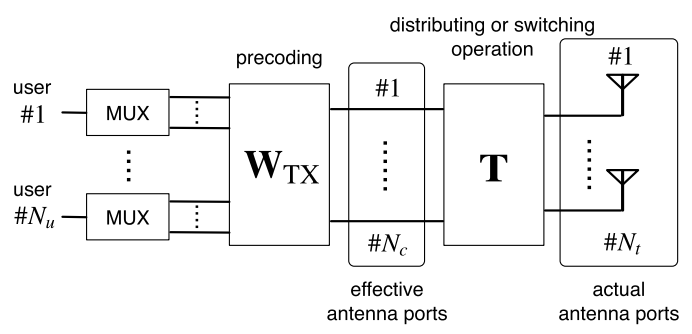

Fig. 7 Transmitter structure.

$$
\mathbf{H}^{(l)} \mathbf{T} \mathbf{W}_{\mathrm{BD}}^{(k)}=\mathbf{O} \text { for } l \neq k .
$$

Therefore, we may regard the effective $\mathrm{BD}$ precoding matrix as $\mathbf{T} \mathbf{W}_{\mathrm{BD}}^{(k)} \in \mathbb{C}^{N_{t} \times N_{w}}$.

In this paper, we apply an eigenmode transmission [21] to each equivalent single-user MIMO channel without IUI. Then, the equivalent channel of the $k$ th user after BD precoding is written as $\tilde{\mathbf{H}}^{(k)} \mathbf{W}_{\mathrm{BD}}^{(k)}=\mathbf{H}^{(k)} \mathbf{T} \mathbf{W}_{\mathrm{BD}}^{(k)}$. The eigenmode precoding matrix $\mathbf{W}_{\mathrm{EM}}^{(k)} \in \mathbb{C}^{N_{w} \times N_{r}}$ is calculated with this equivalent channel $\mathbf{H}^{(k)} \mathbf{T} \mathbf{W}_{\mathrm{BD}}^{(k)}$. The total transmit precoding matrix combining $\mathrm{BD}$ and eigenmode transmission is expressed as

$$
\mathbf{W}_{\mathrm{TX}}^{(k)}=\mathbf{W}_{\mathrm{BD}}^{(k)} \mathbf{W}_{\mathrm{EM}}^{(k)} \mathbf{A}^{(k)},
$$

where $\left(\mathbf{A}^{(k)}\right)^{2}$ is an $N_{r}$-dimensional diagonal matrix for a transmit power control derived from the water-filling theorem. Note that each column vector of $\mathbf{W}_{\mathrm{BD}}^{(k)}$ and $\mathbf{W}_{\mathrm{EM}}^{(k)}$ is normalized. In addition, the total transmit power is set to one and thus the transmit power for each user becomes $1 / N_{u}$.

The whole transmitter structure including the connection matrix is expressed as shown in Fig. 7.

\subsection{Achievable Sum Throughput Evaluation}

In the paper, we evaluate the system performance using achievable sum throughput of the multiuser MIMO system with $\mathrm{BD}$. The received signal vector for the $k$ th user becomes

$$
\mathbf{y}^{(k)}(t)=\mathbf{H}^{(k)} \mathbf{T} \sum_{l=1}^{N_{u}} \mathbf{W}_{\mathrm{TX}}^{(l)} \mathbf{s}^{(l)}(t)+\mathbf{z}^{(k)}(t),
$$

where $\mathbf{s}^{(k)}(t)$ is the transmit signal vector for the user $k$ at the time index $t$ and $\mathbf{z}^{(k)}(t)$ is the noise vector composed of complex Gaussian noise.

Defining an equivalent channel matrix considering the total transmit precoding matrix as $\mathbf{H}_{\mathrm{E}}^{(k, l)}=\mathbf{H}^{(k)} \mathbf{T} \mathbf{W}_{\mathrm{TX}}^{(l)}(l=$ $1,2, \ldots, N_{u}$ ), we can rewrite Eq. (17) as

$$
\mathbf{y}^{(k)}(t)=\sum_{l=1}^{N_{u}} \mathbf{H}_{\mathrm{E}}^{(k, l)} \mathbf{s}^{(l)}(t)+\mathbf{z}^{(k)}(t) .
$$

The achievable sum throughput $\Gamma[\mathrm{bps} / \mathrm{Hz}]$ considering the IUI [22] is expressed as

$$
\Gamma=\sum_{k=1}^{N_{u}} \log _{2} \operatorname{det}\left(\mathbf{I}_{N_{r}}+\left(\mathbf{R}_{\mathrm{NI}}^{(k)}\right)^{-1} \mathbf{H}_{\mathrm{E}}^{(k, k)}\left(\mathbf{H}_{\mathrm{E}}^{(k, k)}\right)^{H}\right),
$$


where $\mathbf{R}_{\mathrm{NI}}^{(k)}$ is the covariance matrix of IUI [22]. $\mathbf{R}_{\mathrm{NI}}^{(k)}$ is given by

$$
\mathbf{R}_{\mathrm{NI}}^{(k)}=\sum_{l=1, l \neq k}^{N_{u}} \mathbf{H}_{\mathrm{E}}^{(k, l)}\left(\mathbf{H}_{\mathrm{E}}^{(k, l)}\right)^{H}+\sigma^{2} \mathbf{I}_{N_{r}},
$$

where $\sigma^{2}$ is the noise power.

In this paper, we assume that effective CSI in Eq. (13) is perfectly estimated in order to focus on effects of signal distributing and signal switching. Thus, no IUI is observed, and so we have

$$
\mathbf{R}_{\mathrm{NI}}^{(k)}=\sigma^{2} \mathbf{I}_{N_{r}} .
$$

\section{Numerical Evaluation}

\subsection{Simulation Environment}

We applied computer simulations to evaluate performance of the O-DAS with two types of transmission methods described above. The simulation parameters are shown in Table 1. The number of RAUs for the DAS and O-DAS is seven as shown in Fig. 8. Thus, the number of antenna elements per RAU is $N_{t} / 7$. Each channel response between antennas except the path loss and shadowing is given as to be i.i.d. quasi-static Rayleigh distribution ${ }^{\dagger}$. The total transmit power and noise level are determined by the cell edge SNR of CAS to be $0 \mathrm{~dB}$ in a single-antenna case considering only path loss ${ }^{\dagger \dagger}$.

The user terminals are uniformly randomly located in the cell, and a complementary cumulative distribution function (CCDF) is obtained by calculating the average sum throughput with Eq. (19) at each position. We evaluated CCDF performances for each transmission method by changing $N_{c}$ and $N_{t}$. Note that $N_{t}$ is limited to 49 at most in the case of $N_{c}=7$ to prevent the port reuse within the RAU. When we use the signal distributing method, the port connection patterns in the connection matrix $\mathbf{T}$ are determined randomly for each trial. The connection matrix in the signal switching method is determined in accordance with a rule based on user locations as described in Sect. 3.2.2.

\subsection{Achievable Sum Throughput Performance}

First, we compare the performance of the O-DAS with that of the conventional DAS. Figure 9 shows the CCDF curves of sum throughputs in the O-DAS case of $N_{t}=21, N_{c}=14$ with signal distributing and signal switching and in the conventional DAS case of $N_{t}=14, N_{c}=14$. For reference,

$\dagger$ The channel correlation between different antennas in the same RAU or base station may not be zero in the actual situations. The specific correlation value depends on the heights of antennas and surrounding buildings. Such conditions may differ between the CAS and DAS. In the paper, we simply assumed the uncorrelated channels as an ideal case.

${ }^{\dagger \dagger}$ These parameters are commonly used in O-DAS, DAS, and CAS.

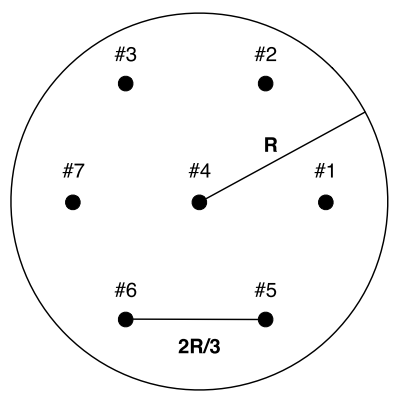

Fig. 8 RAU positions. R denotes the cell radius.

Table 1 Simulation parameters.

\begin{tabular}{|c|c|}
\hline Number of remote antenna units & 7 \\
\hline Number of TX antennas $N_{t}$ & $7,14,21, \ldots, 70$ \\
\hline Number of users $N_{u}$ & 2 \\
\hline Number of RX antennas $N_{r}$ & 3 \\
\hline Channel statistics & Quasi-static Rayleigh fading \\
\hline Path loss constant $\alpha$ & 3.5 \\
\hline Standard deviation of shadowing $\sigma_{s}$ & $7 \mathrm{~dB}$ \\
\hline $\begin{array}{c}\text { Number of trials for sum } \\
\text { throughput (fast fading) averaging }\end{array}$ & 300 \\
\hline $\begin{array}{c}\text { Number of trials (locations) for } \\
\text { CCDF calculation }\end{array}$ & 30000 \\
\hline
\end{tabular}

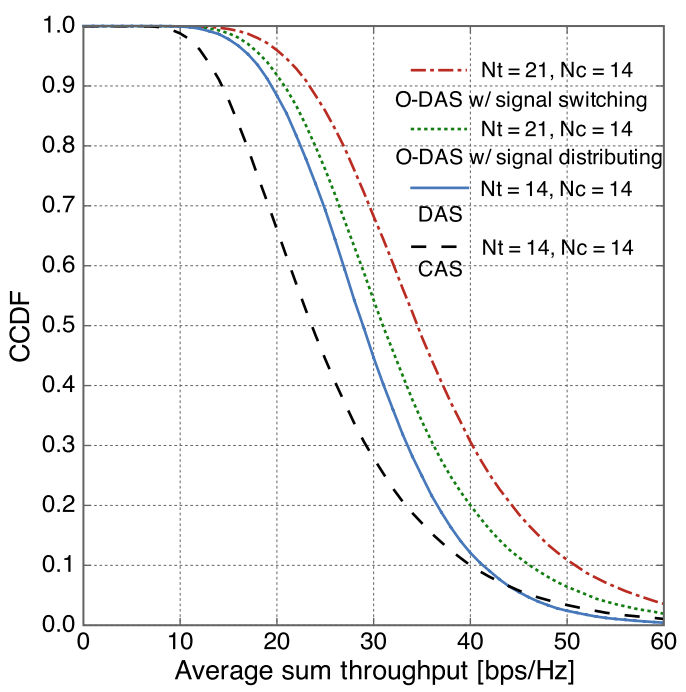

Fig. 9 CCDF of average sum throughput $\left(N_{c}=14\right)$.

we also show the performance in the CAS case of $N_{t}=14$, $N_{c}=14$. All above cases use the same number of pilot sequences $\left(N_{c}=14\right)$. The performances in the O-DAS cases are improved in the whole range, and the gain becomes larger, especially in the large sum throughput region, than in the DAS case. This result clearly indicates that it is effective to increase the number of transmit antennas at each RAU even when pilot resources are limited.

Next, let us discuss the relationship between the number of transmit antennas and pilot sequences applying signal distributing and signal switching. Here, we use $10 \%$ value 


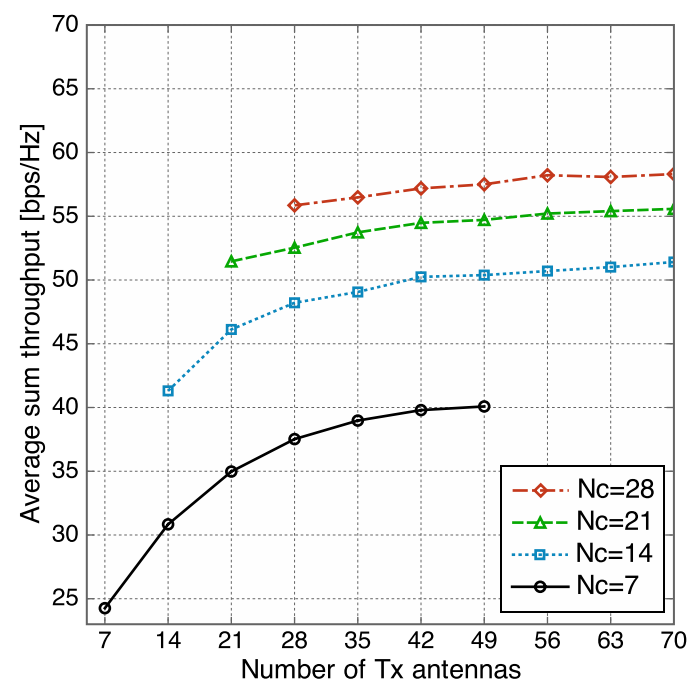

Fig. 10 Effect of signal distributing on average sum throughput performance $(10 \%$ value in $\mathrm{CCDF})$

of sum throughput (near peak throughput) to compare performances.

Figure 10 shows the performance with signal distributing. Note that the leftmost point for each curve corresponds to the case using the same number of transmit antennas and pilot sequences. That is, it represents the performance of the DAS case. The sum throughput monotonically increases as the number of transmit antennas increases regardless of the number of pilot sequences. However, the improvement becomes very small when $N_{t}$ is much larger than $N_{c}$ or when $N_{c}$ is large enough. For example, the gain of increasing $N_{t}$ from 28 to 70 when $N_{c}=28$ is only about $2.46 \mathrm{bps} / \mathrm{Hz}$.

To discuss the performance difference, we investigate the eigenvalue distribution for the cases achieving sum throughput equal to or higher than $10 \%$ value. Here, the maximum number of substreams for each user terminal is three because it has three receive-antennas. Then, we can use the third largest eigenvalue to evaluate the possibility of full-rank transmission. Hence, we focus on only the third largest eigenvalue in the rest of this section.

Figure 11 shows the cumulative distribution function (CDF) curves of the third largest eigenvalue based on the equivalent channel matrix $\mathbf{H}^{(k)} \mathbf{T} \mathbf{W}_{\mathrm{BD}}^{(k)}$. The eigenvalue is greatly improved by increasing the number of transmit antennas in the case of $N_{c}=7$. In contrast, the improvement is small in the case of $N_{c}=28$ even when the number of transmit antennas is increased up to $N_{t}=70$. Here, the large improvement in the third eigenvalues means that the number of effective spatially-multiplexed streams becomes larger. This is closely related with the sum throughput performances. Thus, we can achieve remarkable improvement in the case of $N_{c}=7$ compared with the case of $N_{c}=28$.

On the other hand, the signal switching method provides a larger improvement as shown in Fig. 12. This improvement is given by the power concentration effect of the

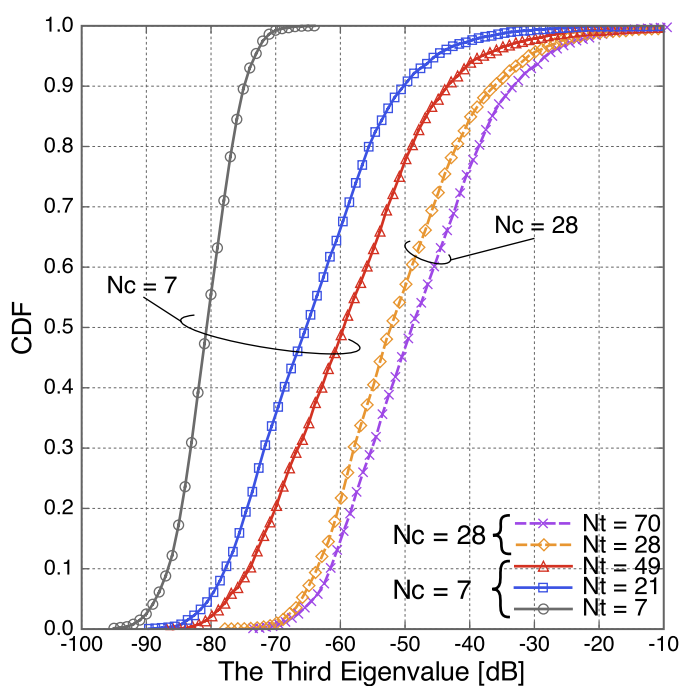

Fig. $11 \mathrm{CDF}$ of the third largest eigenvalue applying signal distributing.

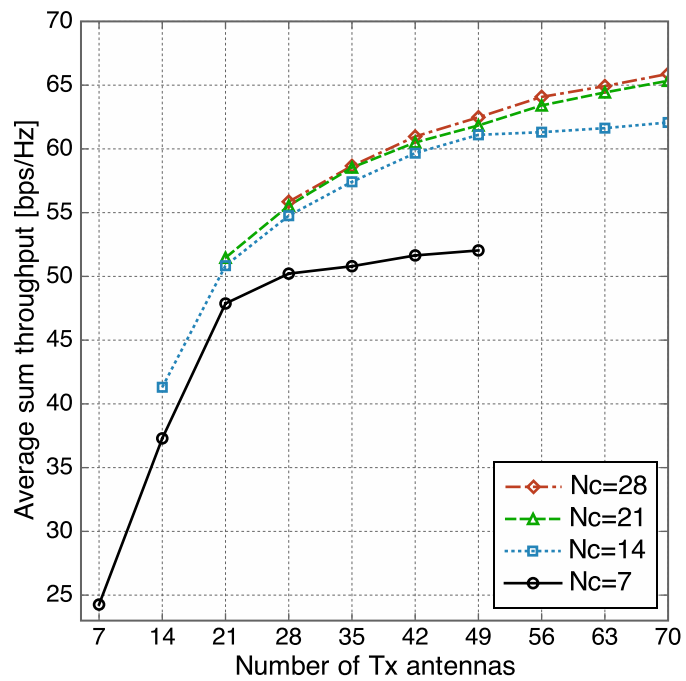

Fig. 12 Effect of signal switching on average sum throughput performance (10\% value in CCDF).

signal switching method ${ }^{\dagger}$. In addition, note that the curves in the case of $N_{c}=14, N_{c}=21$, and $N_{c}=28$ show similar performances. Here, let us check the CDF of the third largest eigenvalue as shown in Fig. 13. In the figure, two curves for $N_{t}=28$ are almost the same. This is the reason the sum throughput performances in the cases of $N_{c}=14$ and $N_{c}=28$ are almost the same. However, the sum throughput in the case of $N_{c}=14$ saturates at $N_{t}=49$. The O-DAS is expected to provide the best performance when two users are near two different RAUs. Then, when $N_{c}=14$, the maximum throughput will be given by selecting seven antennas per RAU. This becomes possible when $N_{t}=49$. According to the selection rule, seven-antenna selection per user, i.e., per RAU, is the upper limit when

${ }^{\dagger}$ In the signal switching method, several RAUs are not activated, whereas in the signal distributing method, all RAUs are activated. 


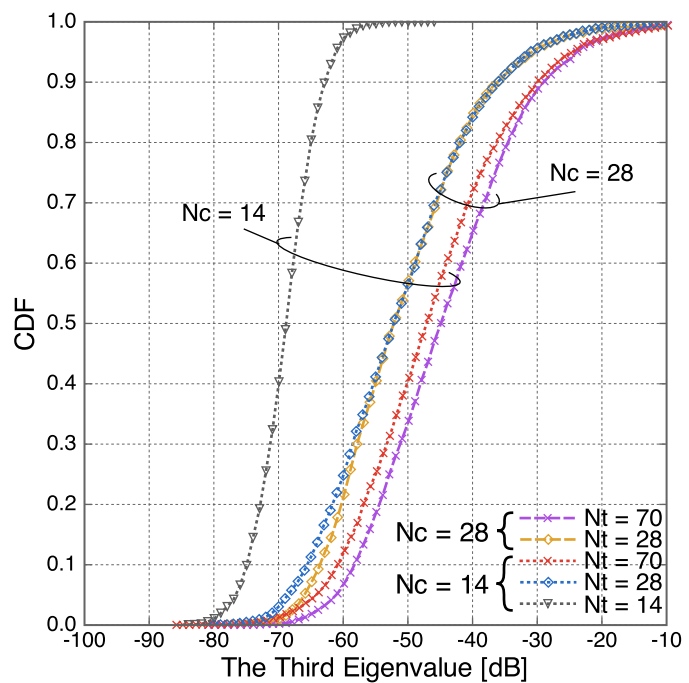

Fig. $13 \mathrm{CDF}$ of the third largest eigenvalue applying signal switching.

$N_{u}=2$. Thus, the gain of increasing the number of antenna elements for $49 \leq N_{t} \leq 70$ is almost the same when $N_{c}=14$.

In contrast, when $N_{c}=28$, the maximum throughput will be given by selecting 14 antennas per RAU. This becomes possible when $N_{t}=98$. Therefore, the sum throughput performance in the case of $N_{c}=28$ still improves for $49 \leq N_{t} \leq 70$. We can confirm this property from the curves of the third largest eigenvalue for $N_{t}=70$ in the cases of $N_{c}=14$ and $N_{c}=28$ as shown in Fig. 13.

Finally, let us show the specific gains of increasing $N_{t}$ with signal distributing and signal switching. The gain of doubling $N_{t}$ with signal distributing is about 6.58 $\mathrm{bps} / \mathrm{Hz}$, and that with signal switching is about $13.03 \mathrm{bps} / \mathrm{Hz}$ for $N_{c}=7$, whereas these gains are reduced to about $2.36 \mathrm{bps} / \mathrm{Hz}$ and $8.22 \mathrm{bps} / \mathrm{Hz}$ for $N_{c}=28$. Thus, we can say that it is particularly effective to apply signal distributing or signal switching to the system with a small number of pilot resources.

\section{Conclusions}

In this paper, we have studied an O-DAS with two transmission methods and evaluated its performance using the achievable sum throughputs given by BD precoding. We improved the sum throughput performance by increasing the number of transmit antennas even when the pilot resources were limited. The effect is especially large in a system with a small number of pilot resources. Although signal switching yields larger improvements than signal distributing, both methods can provide a visible gain in the sum throughput performance. Therefore, we may say that which method we should adopt depends only on the availability of user location information. The optimality of connection matrices for the signal distributing and signal switching should be discussed in further studies.

\section{References}

[1] Y. Nakanishi, T. Nishimura, T. Ohgane, Y. Ogawa, Y. Ohwatari, and Y. Kishiyama, "Antenna splitting in a distributed antenna system for a multiuser MIMO transmission," Proc. IEEE PIMRC'14, Sept. 2014.

[2] "5G radio access: Requirements, concept and technologies," DOCOMO 5G White Paper, NTT DOCOMO, July 2014.

[3] E. Telatar, "Capacity of multi-antenna gaussian channels," Eur. Trans. Telecommun., vol.10, no.6, pp.585-595, Nov./Dec. 1999.

[4] Q.H. Spencer, C.B. Peel, A.L. Swindlehurst, and M. Haardt, "An introduction to the multi-user MIMO downlink," IEEE Commun. Mag., vol.42, no.10, pp.60-67, Oct. 2004.

[5] D. Gesbert, M. Kountouris, R.H. Heath, Jr., C.-B. Chae, and T. Salzer, "Shifting the MEMO paradigm," IEEE Signal Process. Mag., vol.24, no.5, pp.36-46, Sept. 2007.

[6] G. Caire and S. Shamai, "On the achievable throughput of a multiantenna Gaussian broadcast channel," IEEE Trans. Inf. Theory, vol.49, no.7, pp.1691-1706, July 2003.

[7] T.L. Marzetta, "Noncooperative cellular wireless with unlimited numbers of base station antennas," IEEE Trans. Wireless Commun., vol.9, no.11, pp.3590-3600, Nov. 2010.

[8] A.A.M. Saleh, A. Rustako, and R. Roman, "Distributed antennas for indoor radio communications," IEEE Trans. Commun., vol.35, no.12, pp.1245-1251, Dec. 1987.

[9] W. Choi and J. Andrews, "Downlink performance and capacity of distributed antenna systems in a multicell environment," IEEE Trans. Wireless Commun., vol.6, no.1, pp.69-73, 2007.

[10] M.V. Clark, T.M. Willis, L.J. Greenstein, A.J. Rustako, Jr., V. Erceg, and R.S. Roman, "Distributed versus centralized antenna arrays in broadband wireless networks," Proc. IEEE VTS 53rd Vehicular Technology Conference, Spring 2001 (Cat. No.01CH37202), pp.33-37, May 2001.

[11] "C-RAN the road towards green RAN," White paper version 3.0, China Mobile Research Institute, Dec. 2013.

[12] F. Rusek, D. Persson, B.K. Lau, E.G. Larsson, T.L. Marzetta, and F. Tufvesson, "Scaling up MIMO: Opportunities and challenges with very large arrays," IEEE Signal Process. Mag., vol.30, no.1, pp.40-60, Jan. 2013.

[13] H.Q. Ngo, E.G. Larsson, and T.L. Marzetta, "Massive MU-MIMO downlink TDD systems with linear precoding and downlink pilots," Proc. 2013 51st Annual Allerton Conference on Communication, Control, and Computing (Allerton), pp.293-298, Oct. 2013.

[14] S.A. Ramprashad and G. Caire, "Cellular vs. network MIMO: A comparison including the channel state information overhead," Proc. 2009 IEEE 20th International Symposium on Personal, Indoor and Mobile Radio Communications, pp.878-884, Sept. 2009.

[15] S.A. Ramprashad, G. Caire, and H.C. Papadopoulos, "Cellular and network MIMO architectures: MU-MIMO spectral efficiency and costs of channel state information," Proc. 2009 Conference Record of the Forty-Third Asilomar Conference on Signals, Systems and Computers, pp.1811-1818, Nov. 2009.

[16] F. Diehm, P. Marsch, and G. Fettweis, "The FUTON prototype: Proof of concept for coordinated multi-point in conjunction with a novel integrated wireless/optical architecture," Proc. 2010 IEEE Wireless Communication and Networking Conference Workshops, pp.1-4, April 2010.

[17] A. Goldsmith, Wireless Communication, Cambridge University Press, 2005.

[18] Q.H. Spencer, A.L. Swindlehurst, and M. Haardt, "Zero-forcing methods for downlink spatial multiplexing in multiuser MIMO channels," IEEE Trans. Signal Process., vol.52, no.2, pp.461-471, 2004.

[19] M. Rim, "Multi-user downlink beamforming with multiple transmit and receive antennas," Electron. Lett., vol.38, no.25, pp.1725-1726, 2002. 
[20] K. Nishimori, T. Hiraguri, and H. Makino, "Transmission rate by user antenna selection for block diagonalization based multiuser MIMO system," IEICE Trans. Commun., vol.E97-B, no.10, pp.2118-2126, Oct. 2014.

[21] K. Miyashita, T. Nishimura, T. Ohgane, Y. Ogawa, Y. Takatori, and K. Cho, "High data-rate transmission with eigenbeam-space division multiplexing (E-SDM) in a MIMO channel," Proc. IEEE 56th Vehicular Technology Conference, vol.3, pp.1302-1306, Sept. 2002.

[22] Yi Song and S.D. Blostein, "MIMO channel capacity in co-channel interference," Proc. 21st Biennial Symposium on Communications, pp.220-224, Kingston, Canada, May 2002.

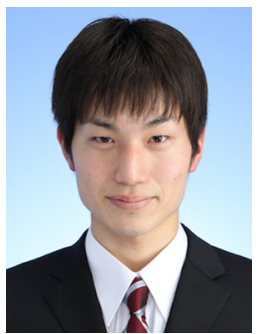

Yuki Nakanishi received a B.E. degree in information science and technology from Hokkaido University, Sapporo, Japan, in 2013. $\mathrm{He}$ is currently a master's course student at the Graduate School of Information Science and Technology, Hokkaido University. His interests are in signal processing for distributed antenna systems. He is a student member of IEEE.

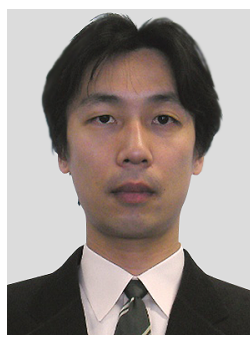

Toshihiko Nishimura received his B.S in physics in 1992, his M.S. in physics in 1994, and his Ph.D. in electronics engineering in 1997 all from Hokkaido University, Sapporo, Japan. He joined the Graduate School of Engineering (presently reorganized as the Graduate School of Information Science and Technology) at Hokkaido University in 1998, where he is currently an assistant professor of the Graduate School of Information Science and Technology. His current research interests are in MIMO systems using smart antenna techniques. Dr. Nishimura received the Young Researchers' Award of IEICE in 2000, the Best Paper Award from IEICE in 2007, and the Best Magazine Paper Award in 2011 from the IEICE Communications Society. He is a member of IEEE.

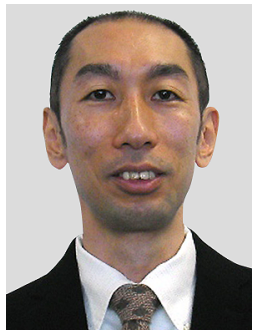

Takeo Ohgane received his B.E., M.E., and $\mathrm{Ph} . \mathrm{D}$. in electronics engineering from Hokkaido University, Sapporo, Japan, in 1984, 1986, and 1994. He was with the Communications Research Laboratory of the Ministry of Posts and Telecommunications from 1986 to 1992 . He was on assignment at the ATR Optical and Radio Communications Research Laboratory from 1992 to 1995 . Since 1995 , he has been with Hokkaido University, where he is an associate professor. He was at the Centre for Communications Research of the University of Bristol, U.K., as a visiting fellow from 2005-2006. His interests are in MIMO signal processing for wireless communications. Dr. Ohgane received the Young Researchers' Award of IEICE in 1990, the IEEE AP-S Tokyo Chapter Young Engineer Award in 1993, the Best Paper Award from IEICE in 2007, and the Best Magazine Paper Award in 2011 from the IEICE Communications Society. He is a member of IEEE.

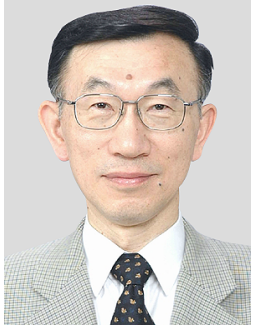

Yasutaka Ogawa received his B.E., M.E., and Ph.D. from Hokkaido University, Sapporo, Japan, in 1973, 1975, and 1978. Since 1979, he has been with Hokkaido University, where he is currently a full professor at the Graduate School of Information Science and Technology. From 1992-1993, he was with the ElectroScience Laboratory of Ohio State University, U.S.A., as a visiting scholar, on leave from Hokkaido University. His interests are in adaptive antennas, mobile communications, superresolution techniques, and MIMO systems. Dr. Ogawa received the Yasujiro Niwa outstanding paper award in 1978, the Young Researchers' Award of IEICE in 1982, the Best Paper Award from IEICE in 2007, the Best Magazine Paper Award in 2011 from the IEICE Communications Society, and the Achievement Award from IEICE in 2014. He is a fellow of IEEE.

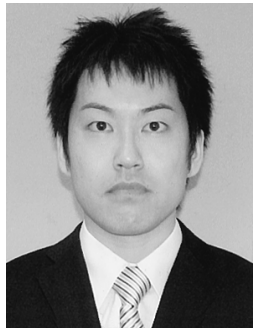

Yusuke Ohwatari received his B.E. in electronics engineering, M.E. in information science and technology, and his Ph.D. in engineering from Hokkaido University, Hokkaido, Japan, in 2005, 2007, and 2013. Since 2007, he has been with the Research Laboratories, NTT DOCOMO, INC., Kanagawa, Japan. His current interest is multiple-input and multipleoutput (MIMO) systems for mobile communication systems. He received the IEEE VTS Japan Chapter Young Researcher's Encouragement Award in 2006, the Best Paper Award from IEICE in 2007, the Young Researchers' Award from IEICE in 2012, and the 2011 IEICE RCS Active Research Award. He is a member of IEEE.

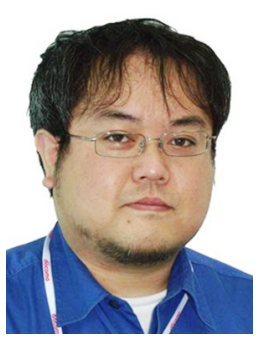

Yoshihisa Kishiyama received his B.E., M.E., and Ph.D. from Hokkaido University, Sapporo, Japan, in 1998, 2000, and 2010. In 2000, he joined NTT DOCOMO, INC. His research interests include physical layer technologies for 3GPP LTE enhancements and future radio access $(5 \mathrm{G})$ concept for efficient use of higher frequency bands. He was a recipient of the IEICE Young Engineer Award in 2004 and the ITU Association of Japan Award in 2012. $\mathrm{He}$ is currently a manager in the Radio Access Development Department of NTT DOCOMO. He is a member of IEEE. 